\title{
On 1-dimensional representations of finite $W$-algebras associated to simple Lie algebras of exceptional type
}

\author{
Simon M. Goodwin, Gerhard Röhrle and Glenn Ubly
}

\begin{abstract}
We consider the finite $W$-algebra $U(\mathfrak{g}, e)$ associated to a nilpotent element $e \in \mathfrak{g}$ in a simple complex Lie algebra $\mathfrak{g}$ of exceptional type. Using presentations obtained through an algorithm based on the PBW-theorem for $U(\mathfrak{g}, e)$, we verify a conjecture of Premet, that $U(\mathfrak{g}, e)$ always has a 1-dimensional representation when $\mathfrak{g}$ is of type $G_{2}, F_{4}, E_{6}$ or $E_{7}$. Thanks to a theorem of Premet, this allows one to deduce the existence of minimal dimension representations of reduced enveloping algebras of modular Lie algebras of the above types. In addition, a theorem of Losev allows us to deduce that there exists a completely prime primitive ideal in $U(\mathfrak{g})$ whose associated variety is the coadjoint orbit corresponding to $e$.
\end{abstract}

\section{Introduction}

Finite $W$-algebras were introduced into the mathematical literature by Premet in [16]. We recall that a finite $W$-algebra $U(\mathfrak{g}, e)$ is a certain finitely generated algebra associated to a reductive Lie algebra $\mathfrak{g}$ and a nilpotent element $e \in \mathfrak{g}$; it is a quantization of the Slodowy slice through the nilpotent orbit of $e$. Recently, there has been much research activity in the representation theory of $U(\mathfrak{g}, e)$; see for example $[\mathbf{2}, \mathbf{3}, \mathbf{9 - 1 2}, \mathbf{1 7}-\mathbf{1 9}]$. However, the fundamental question of the existence of a 1-dimensional representation of $U(\mathfrak{g}, e)$ remains open in general. Indeed, it was only recently proved by Premet in [18, Corollary 1.1] that $U(\mathfrak{g}, e)$ always has finite-dimensional representations; alternative proofs Losev [10, Theorem 1.2.2(viii)] and Ginzburg [9, Theorem 4.5.2]. It was conjectured by Premet in [17, Conjecture 3.1] that there is always a 1-dimensional $U(\mathfrak{g}, e)$-module; this conjecture has now been verified for $\mathfrak{g}$ of classical type by Losev in [10, Theorem 1.2.3]. Further, the conjecture was reduced to the case where $e$ is rigid by Premet in [19, Theorem 1.1]; we recall that $e$ is rigid if the nilpotent orbit of $e$ cannot be obtained through Lusztig-Spaltenstein induction [14] from a nilpotent orbit in a Levi subalgebra of $\mathfrak{g}$.

In this article we consider Premet's conjecture in the case where $\mathfrak{g}$ is of exceptional type: our main result is the following theorem.

TheOrem 1.1. Let $\mathfrak{g}$ be a simple Lie algebra over $\mathbb{C}$ of type $G_{2}, F_{4}, E_{6}$ or $E_{7}$, and let $e \in \mathfrak{g}$ be nilpotent. Then there exists a 1-dimensional representation of the finite $W$-algebra $U(\mathfrak{g}, e)$.

Our approach is computational and based on the Poincaré-Birkhoff-Witt (PBW) theorem for finite $W$-algebras; see [16, Theorem 4.6] along with the above mentioned theorem of Premet reducing to the case where $e$ is rigid. We have developed an algorithm that determines an explicit presentation of a given finite $W$-algebra which we have used for the cases $\mathfrak{g}$ of type $G_{2}, F_{4}, E_{6}$ or $E_{7}$ and $e$ rigid. From these presentations, it is straightforward to determine all 1-dimensional representations of $U(\mathfrak{g}, e)$; in fact, the number of 1-dimensional representations is either one or two, see Table 1. We remark that it is possible to determine the 1-dimensional

Received 17 June 2009; revised 17 March 2010.

2000 Mathematics Subject Classification $17 \mathrm{~B} 37$ (primary), 17B10, $81 \mathrm{R} 05$ (secondary). 
representations without calculating a full presentation, which we have done in a number of cases when $\mathfrak{g}$ is of type $E_{7}$; see Proposition 3.5 and $\S 5$ for more details.

Our results taken with the work of Losev and Premet mean that Premet's conjecture is reduced to the cases where $\mathfrak{g}$ is of type $E_{8}$ and $e$ lies in a rigid orbit of $\mathfrak{g}$. Our methods allow us to prove the existence of 1-dimensional $U(\mathfrak{g}, e)$-modules for some rigid $e$ in the case where $\mathfrak{g}$ is of type $E_{8}$; at present it is computationally unfeasible to deal with those $e$ with large height, see Remark 5.1 for more details. Also, after this paper was written, Losev announced in [13] an alternative approach to finding the 1-dimensional representations of $U(\mathfrak{g}, e)$ based on the highest weight theory from [2].

We now discuss how results of Premet provide an application of Theorem 1.1 to the representation theory of modular Lie algebras, which we state in Theorem 1.2. It is necessary to introduce some notation.

Let $G$ be a simple simply connected algebraic group over $\mathbb{C}$. Let $\mathfrak{g}$ be the Lie algebra of $G$ and let $\mathfrak{g}_{\mathbb{Z}}$ be a Chevalley $\mathbb{Z}$-form of $\mathfrak{g}$. Let $p$ be a prime that is assumed to be good for $G$ and let $\mathbb{k}$ be the algebraic closure of $\mathbb{F}_{p}$. Let $\mathfrak{g}_{\mathbb{k}}=\mathfrak{g}_{\mathbb{Z}} \otimes \mathbb{k}$ and let $\xi \in \mathfrak{g}_{\mathrm{k}}^{*}$. The reduced enveloping algebra corresponding to $\xi$ is denoted $U_{\xi}\left(\mathfrak{g}_{\mathrm{k}}\right)$. We write $d_{\xi}$ for half the dimension of the coadjoint orbit of $\xi$.

Given a nilpotent orbit in $\mathfrak{g}$ it is possible to choose a representative $e \in \mathfrak{g}_{\mathbb{Z}}$ such that we can find an $\mathfrak{s l}_{2}$-triple $(e, h, f)$ contained in $\mathfrak{g}_{\mathbb{Z}}$, see $\S 4.2$. Let $\kappa$ be the Killing form on $\mathfrak{g}$ and assume that $p$ is such that it does not divide $\kappa(e, f)$. Then we can normalize $\kappa$ to obtain the bilinear form $(\cdot, \cdot)$ with $(e, f)=1$, which can be viewed as a bilinear form on both $\mathfrak{g}$ and $\mathfrak{g}_{\mathbb{k}}$. We may view $e$ both as an element of $\mathfrak{g}$ and $\mathfrak{g}_{\mathfrak{k}}$, and therefore view $\chi$ defined by $\chi(x)=(x, e)$ both as an element of $\mathfrak{g}^{*}$ and $\mathfrak{g}_{\mathbb{k}}^{*}$.

The Kac-Weisfeiler conjecture, proved by Premet in [15], says that the dimension of a $U_{\xi}\left(\mathfrak{g}_{\mathbb{k}}\right)$-module is divisible by $p^{d_{\xi}}$. The existence of a $U_{\xi}\left(\mathfrak{g}_{\mathbb{k}}\right)$-module with dimension equal to $p^{d_{\xi}}$ is an open problem; see $[\mathbf{1 5}, \S 4.4]$. Thanks to the Kac-Weisfeiler theorem, or its generalization [6, Theorem 3.2] due to Friedlander and Parshall, there is a reduction to the case of nilpotent $\xi$.

In [19, Theorem 1.4], Premet proves that the existence of a 1-dimensional $U(\mathfrak{g}, e)$-module implies existence of a representation of $U_{\chi}\left(\mathfrak{g}_{\mathrm{k}}\right)$ of dimension $p^{d_{\chi}}$ for $p$ sufficiently large. As a consequence of Premet's and Losev's results, the existence of a minimal dimension representation of $U_{\xi}\left(\mathfrak{g}_{\mathrm{k}}\right)$ is now proved for $\mathfrak{g}$ of classical type and $p$ sufficiently large; see [19, Corollary 1.1]. We can deduce the following from Theorem 1.1.

THEOREM 1.2. Let $\mathfrak{g}_{\mathrm{k}}$ be a simple Lie algebra over $\mathbb{k}$ of type $G_{2}, F_{4}, E_{6}$ or $E_{7}$. Assume that $p \gg 0$ and let $\xi \in \mathfrak{g}_{\mathbb{k}}^{*}$. Then the reduced enveloping algebra $U_{\xi}\left(\mathfrak{g}_{\mathbb{k}}\right)$ has a simple module of dimension $p^{d_{\xi}}$, where $d_{\xi}$ is half the dimension of the coadjoint orbit of $\xi$.

The restriction in [19, Theorem 1.4] leading to the condition that $p$ is sufficiently large stems from a number of rationality assumptions in the construction of $U(\mathfrak{g}, e)$ made in [19, Section 2]; in particular, that $U(\mathfrak{g}, e)$ is defined over $\mathbb{Z}\left[d^{-1}\right]$, but only for $d$ a sufficiently large integer. As discussed in [19, Remark 2.2], this bound can be lowered by knowledge of explicit presentations of $U(\mathfrak{g}, e)$. In the cases where $\mathfrak{g}$ is of type $G_{2}, F_{4}$ and $E_{6}$, we are able to give an explicit bound, which just requires $p$ to be good and not to divide all $\kappa\left(e_{0}, f_{0}\right)$, where $\left(e_{0}, h_{0}, f_{0}\right)$ is an $\mathfrak{s l}_{2}$-triple in a Levi subalgebra of $\mathfrak{g}$ in which $e_{0}$ is rigid; see Remark 5.2 for more details.

Next we discuss how Theorem 1.1 implies existence of completely prime primitive ideals, through Skryabin's equivalence and a result of Losev. This is stated in Theorem 1.3 below.

We recall that Skryabin's equivalence gives an equivalence of categories between the category of $U(\mathfrak{g}, e)$-modules and a certain category of generalized Whittaker modules; see $[\mathbf{2 0}]$ or $[\mathbf{7}$, Theorem 6.1]. For a $U(\mathfrak{g}, e)$-module $M$, we write $\widehat{M}$ for the Whittaker $U(\mathfrak{g})$-module obtained through this equivalence, and $\mathcal{I}_{M}$ for the annihilator of $\widehat{M}$ in $U(\mathfrak{g})$. Premet proved in $[\mathbf{1 6}$, Theorem 3.1] that the associated variety $\mathcal{V} \mathcal{A}\left(\mathcal{I}_{M}\right)$ of $\mathcal{I}_{M}$ contains the coadjoint orbit $G \cdot \chi$ 
and that $\mathcal{V} \mathcal{A}\left(\mathcal{I}_{M}\right)=G \cdot \chi$ if and only if $M$ is finite-dimensional. Further, it was conjectured in [17, Conjecture 3.1] that if $M$ is 1-dimensional, then $\mathcal{I}_{M}$ is completely prime; this conjecture was verified by Losev in [10, Proposition 3.4.6]. Therefore, we can deduce the following from Theorem 1.1.

Theorem 1.3. Let $\mathfrak{g}$ be a simple Lie algebra over $\mathbb{C}$ of type $G_{2}, F_{4}, E_{6}$ or $E_{7}$. Let $e \in \mathfrak{g}$ be nilpotent and let $\chi=(e, \cdot) \in \mathfrak{g}^{*}$. Then there is a completely prime primitive ideal of $U(\mathfrak{g})$ whose associated variety is $G \cdot \chi$.

We give a brief outline of the structure of the paper. First we recall the definition of the finite $W$-algebra $U(\mathfrak{g}, e)$ and state a version of the PBW theorem for $U(\mathfrak{g}, e)$ in $\S 2$. Next, in $\S 3$ we use the PBW theorem to give presentations of $U(\mathfrak{g}, e)$ and show, in Theorem 3.2, that some of the relations are superfluous. Our algorithm is explained in $\S 4$ and we discuss the results obtained from it in $\S 5$. Finally, in $\S 6$ we present an example of how the algorithm works for $\mathfrak{g}$ of type $G_{2}$ and $e$ a short root vector.

\section{Preliminaries}

We begin by giving the notation that we require, and recalling the definition of the finite $W$-algebra $U(\mathfrak{g}, e)$. There are at present three equivalent definitions of finite $W$-algebras in the literature. Here we only consider the Whittaker model definition introduced in [16]: this was proved to be equivalent to the definition via BRST cohomology in [4] and to the definition via Fedosov quantization in [10, Corollary 3.3.3].

\subsection{Notation}

Let $G$ be a simple simply connected algebraic group over $\mathbb{C}$ and let $\mathfrak{g}$ be the Lie algebra of $\mathfrak{g}$. Let $e \in \mathfrak{g}$ be a nilpotent element and let $(e, h, f)$ be an $\mathfrak{s l}_{2}$-triple in $\mathfrak{g}$. Let $(\cdot, \cdot)$ be a non-degenerate symmetric invariant bilinear form on $\mathfrak{g}$ normalized so that $(e, f)=1$. Define $\chi \in \mathfrak{g}^{*}$ by $\chi(x)=(e, x)$ for $x \in \mathfrak{g}$. The Dynkin grading $\mathfrak{g}=\bigoplus_{j \in \mathbb{Z}} \mathfrak{g}(j)$ of $\mathfrak{g}$ is defined by $\mathfrak{g}(j)=\{x \in \mathfrak{g} \mid[h, x]=j x\}$. We write $\mathfrak{g}^{e}$ for the centralizer of $e$ in $\mathfrak{g}$.

Let $\mathfrak{t}^{e}$ be a Cartan subalgebra of $\mathfrak{g}^{e} \cap \mathfrak{g}(0)$, and let $\mathfrak{t}$ be a Cartan subalgebra of $\mathfrak{g}$ containing $\mathfrak{t}^{e}$. We write $\Phi \subseteq \mathfrak{t}^{*}$ for the root system of $\mathfrak{g}$ with respect to $\mathfrak{t}$ and $\Pi$ for a set of simple roots. Recall that the restricted root system $\Phi^{e}$ is defined by $\Phi^{e}=\left\{\left.\alpha\right|_{\mathfrak{t}^{e}} \mid \alpha \in \Phi\right\} \subseteq\left(\mathfrak{t}^{e}\right)^{*}$; see [1, Sections 2 and 3]. We have the $\mathfrak{t}^{e}$-weight space decomposition $\mathfrak{g}=\mathfrak{g}_{0} \oplus \bigoplus_{\alpha \in \Phi^{e}} \mathfrak{g}_{\alpha}$ of $\mathfrak{g}$, where $\mathfrak{g}_{\alpha}=\left\{x \in \mathfrak{g} \mid[t, x]=\alpha(t) x\right.$ for all $\left.t \in \mathfrak{t}^{e}\right\}$ for $\alpha \in \Phi^{e} \cup\{0\}$.

\subsection{Definition of $U(\mathfrak{g}, e)$}

For $x, y \in \mathfrak{g}(-1)$, let $\langle x, y\rangle=\chi([x, y])$, so that $\langle\cdot, \cdot\rangle$ defines a non-degenerate alternating bilinear form on $\mathfrak{g}(-1)$. Choose a Lagrangian subspace $\mathfrak{g}(-1)^{0}$ of $\mathfrak{g}(-1)$ with respect to $\langle\cdot, \cdot\rangle$ and define the nilpotent subalgebra

$$
\mathfrak{m}=\mathfrak{g}(-1)^{0} \oplus \bigoplus_{i \leqslant-2} \mathfrak{g}(i)
$$

of $\mathfrak{g}$. It is straightforward to check that $\chi$ restricts to a character of $\mathfrak{m}$, so we can consider the 1-dimensional $U(\mathfrak{m})$-module $\mathbb{C}_{\chi}$ and the induced module $Q_{\chi}=U(\mathfrak{g}) \otimes_{U(\mathfrak{m})} \mathbb{C}_{\chi}$. The finite $W$-algebra associated to $\mathfrak{g}$ and $e$ is defined to be the endomorphism algebra

$$
U(\mathfrak{g}, e)=\operatorname{End}_{U(\mathfrak{g})}\left(Q_{\chi}\right)^{\text {op }} .
$$

Let $I_{\chi}$ be the left ideal of $U(\mathfrak{g})$ generated by all $x-\chi(x)$ for $x \in \mathfrak{m}$. The PBW theorem for $U(\mathfrak{g})$ implies that $Q_{\chi}$ identifies with $U(\mathfrak{g}) / I_{\chi}$ as a vector space. Through this identification we 
get an isomorphism between $U(\mathfrak{g}, e)$ and the space of $\mathfrak{m}$-invariants of $U(\mathfrak{g}) / I_{\chi}$ :

$$
\left\{u+I_{\chi} \in U(\mathfrak{g}) / I_{\chi} \mid[x, u] \in I_{\chi} \text { for all } x \in \mathfrak{m}\right\} .
$$

We use these identifications throughout the sequel.

Thanks to [17, Lemma 2.4], there is an embedding $\mathfrak{t}^{e} \hookrightarrow U(\mathfrak{g}, e)$. We identify $\mathfrak{t}^{e}$ with its image in $U(\mathfrak{g}, e)$, so we have an adjoint action of $\mathfrak{t}^{e}$ on $U(\mathfrak{g}, e)$. The weights of this action lie in $\mathbb{Z} \Phi^{e} \subseteq\left(\mathfrak{t}^{e}\right)^{*}$.

\subsection{The $P B W$ theorem for $U(\mathfrak{g}, e)$}

In Theorem 2.1 below we state a version of the PBW theorem for $U(\mathfrak{g}, e)$. To state this explicitly we need to pick a suitable basis of $\mathfrak{g}$.

Define the parabolic subalgebra $\mathfrak{p}=\bigoplus_{j \geqslant 0} \mathfrak{g}(j)$ of $\mathfrak{g}$; note that we have the inclusion $\mathfrak{g}^{e} \subseteq \mathfrak{p}$. Let $x_{1}, \ldots, x_{r}$ be a basis of $\mathfrak{g}^{e}$ and extend this to a basis $x_{1}, \ldots, x_{m}$ of $\mathfrak{p}$; we assume that $x_{i}=e$ for some $i \in\{1, \ldots, r\}$. The form $\langle\cdot, \cdot\rangle$ is $\mathfrak{t}^{e}$-invariant, that is $\langle[x, t], y\rangle=\langle x,[t, y]\rangle$ for $x, y \in \mathfrak{g}(-1)$ and $t \in \mathfrak{t}^{e}$, so we may choose a Witt basis $z_{1}, \ldots, z_{s}, z_{1}^{*}, \ldots, z_{s}^{*}$ of $\mathfrak{g}(-1)$ consisting of $\mathfrak{t}^{e}$-weight vectors. We choose $\mathfrak{g}(-1)^{0}$ to have basis $z_{1}^{*}, \ldots, z_{s}^{*}$, and set $x_{m+i}=z_{i}$ and $x_{m+s+i}=z_{i}^{*}$ for $i=1, \ldots, s$. Next we choose a basis $x_{m+2 s+1}, \ldots, x_{m+2 s+s^{\prime}}$ of $\mathfrak{g}(-2)$ as follows. It is clear that $\left.\operatorname{ker} \chi\right|_{\mathfrak{g}(-2)}$ is $\mathfrak{t}^{e}$-stable, so we choose $x_{m+2 s+1}, \ldots, x_{m+2 s+s^{\prime}-1}$ to be $\mathfrak{t}^{e}$-weight vectors forming a basis of ker $\left.\chi\right|_{\mathfrak{g}(-2)}$ and $x_{m+2 s+s^{\prime}}=f$. Finally, we extend to a basis $x_{1}, \ldots, x_{n}$ of all of $\mathfrak{g}$.

It is clear that we can choose all of the elements of our basis to be weight vectors for $\mathfrak{t}^{e}$ and eigenvectors for ad $h$. We let $n_{i} \in \mathbb{Z}$ and $\beta_{i} \in \Phi^{e}$ be such that $x_{i} \in \mathfrak{g}\left(n_{i}\right) \cap \mathfrak{g}_{\beta_{i}}$ for $i=1, \ldots, n$.

Let $\left\{e_{\alpha} \mid \alpha \in \Phi\right\} \cup\left\{h_{\alpha} \mid \alpha \in \Pi\right\}$ be a Chevalley basis of $\mathfrak{g}$. Let $\mathfrak{g}_{\mathbb{Z}}$ be the corresponding $\mathbb{Z}$-form on $\mathfrak{g}$ and let $\mathfrak{g}_{\mathbb{Q}}=\mathfrak{g}_{\mathbb{Z}} \otimes_{\mathbb{Z}} \mathbb{Q}$. It is well-known that the $G$-orbit of $e$ intersects $\mathfrak{g}_{\mathbb{Z}}$, so we can assume that $e \in \mathfrak{g}_{\mathbb{Z}}$. Then it is easy to see that we can choose our basis elements $x_{1}, \ldots, x_{n} \in \mathfrak{g}_{\mathbb{Q}}$.

From the PBW theorem for $U(\mathfrak{g})$ we see that a basis of $Q_{\chi}$ is given by the cosets $x^{\mathbf{a}}+I_{\chi}=x_{1}^{a_{1}} \ldots x_{m+s}^{a_{m+s}}+I_{\chi}$ for $\mathbf{a}=\left(a_{1}, \ldots, a_{m+s}\right) \in \mathbb{Z}_{\geqslant 0}^{m+s}$. For $\mathbf{a} \in \mathbb{Z}_{\geqslant 0}^{m+s}$, we define

$$
|\mathbf{a}|=\sum_{i=1}^{m+s} a_{i} \quad \text { and } \quad|\mathbf{a}|_{e}=\sum_{i=1}^{m+s} a_{i}\left(n_{i}+2\right) .
$$

This allows us to define the Kazhdan filtration on $Q_{\chi}$ by declaring that $x^{\mathbf{a}}+I_{\chi}$ has filtered degree $|\mathbf{a}|_{e}$. This restricts to the Kazhdan filtration on $U(\mathfrak{g}, e)$ and we write $F_{i} U(\mathfrak{g}, e)$ for the $i$ th filtered part of the Kazhdan filtration on $U(\mathfrak{g}, e)$. Given an element $u \in U(\mathfrak{g}, e)$, we say that $u$ has Kazhdan degree $i$ to mean $u \in F_{i} U(\mathfrak{g}, e)$, not necessarily assuming that $i$ is minimal.

The PBW theorem for $U(\mathfrak{g}, e)$ is sometimes stated in terms of the associated graded algebra $\operatorname{gr} U(\mathfrak{g}, e)$ for the Kazhdan filtration; see for example [7, Theorem 4.1]. For our purposes the following more explicit version is more convenient. The statement combines [15, Theorem 4.1] and $[\mathbf{1 7}$, Lemma 2.2]. The uniqueness statements are not given in the references, but they are straightforward to deduce.

TheOREm 2.1. Let $x_{1}, \ldots, x_{n}$ be a basis of $\mathfrak{g}$ as above. Then the following hold.

(1) There is a set of generators for $U(\mathfrak{g}, e)$ given by

$$
\Theta_{i}=\left(x_{i}+\sum_{|\mathbf{a}|_{e} \leqslant n_{i}+2} \lambda_{\mathbf{a}}^{i} x^{\mathbf{a}}\right)+I_{\chi},
$$

for $i=1, \ldots, r$, where the coefficients $\lambda_{\mathbf{a}}^{i} \in \mathbb{Q}$ are zero when $a_{r+1}=\ldots=a_{m+s}=0$, or if $|\mathbf{a}|_{e}=n_{i}+2$ and $|\mathbf{a}|=1$. The coefficients $\lambda_{\mathbf{a}}^{i}$ are uniquely determined by the choice of ordered basis $x_{1}, \ldots, x_{n}$ of $\mathfrak{g}$ and the above vanishing conditions.

(2) The $\Theta_{i}$ are weight vectors for $\mathfrak{t}^{e}$ with weight $\beta_{i}$.

(3) The monomials $\Theta^{\mathbf{a}}=\Theta_{1}^{a_{1}} \ldots \Theta_{r}^{a_{r}}$ with $\mathbf{a} \in \mathbb{Z}_{\geqslant 0}^{r}$ form a $P B W$ basis of $U(\mathfrak{g}, e)$. 
(4) We have $\left[\Theta_{i}, \Theta_{j}\right] \in F_{n_{i}+n_{j}+2} U(\mathfrak{g}, e)$. Moreover, if $\left[x_{i}, x_{j}\right]=\sum_{k=1}^{r} \mu_{i j}^{k} x_{k}$ in $\mathfrak{g}^{e}$, then

$$
\left[\Theta_{i}, \Theta_{j}\right]=\sum_{k=1}^{r} \mu_{i j}^{k} \Theta_{k}+q_{i j}\left(\Theta_{1}, \ldots, \Theta_{r}\right) \bmod F_{n_{i}+n_{j}} U(\mathfrak{g}, e),
$$

where $q_{i j}$ is a polynomial with coefficients in $\mathbb{Q}$, and zero constant and linear terms.

\section{Removing relations}

Theorem 2.1 allows one to determine a presentation of $U(\mathfrak{g}, e)$, as in [18, Lemma 4.1], from which one can work out all 1-dimensional representations of $U(\mathfrak{g}, e)$. However, this presentation involves a large number of commutator relations, so is rather laborious to calculate. In Theorem 3.2 we show that fewer relations suffice to obtain a presentation of $U(\mathfrak{g}, e)$. Further, in Proposition 3.5 we show that if we are only interested in determining the 1-dimensional representations of $U(\mathfrak{g}, e)$, then we need to consider even fewer relations.

In the following lemma, which is required for the proof of Theorem 3.2, we use the notation introduced in $\S 2.3$ and Theorem 2.1.

Lemma 3.1. Suppose that $\mathfrak{g}^{e}$ is generated by $x_{1}, \ldots, x_{b}$ for $b \leqslant r$. Then $U(\mathfrak{g}, e)$ is generated by $\Theta_{1}, \ldots, \Theta_{b}$.

Proof. We may assume that $x_{b+1}, \ldots, x_{r}$ are chosen so that $n_{b+1} \leqslant \ldots \leqslant n_{r}$. The assumption that $x_{1}, \ldots, x_{b}$ generate $\mathfrak{g}^{e}$ implies that there exists $i, j \leqslant b$ such that $\left[x_{i}, x_{j}\right]$ is in $\mathfrak{g}\left(n_{b+1}\right)$, but does not lie in the subspace of $\mathfrak{g}^{e}$ spanned by $x_{1}, \ldots, x_{b}$. Now we can assume that we picked our basis of $\mathfrak{g}^{e}$ with $x_{b+1}=\left[x_{i}, x_{j}\right]$. Similar arguments show that for $k>b$ we can assume that

$$
x_{k}=\sum_{i, j<k} \nu_{i j}^{k}\left[x_{i}, x_{j}\right]
$$

where $\nu_{i j}^{k} \in \mathbb{Q}$.

To prove the lemma, it suffices to show that $\Theta_{i}$ lies in the subalgebra $W$ of $U(\mathfrak{g}, e)$ generated by $\Theta_{1}, \ldots, \Theta_{b}$ for $i=b+1, \ldots, r$. Suppose that we have shown that $\Theta_{b+1}, \ldots, \Theta_{k-1} \in W$; in particular, this means that $F_{n_{k}+1} U(\mathfrak{g}, e) \subseteq W$. From Theorem 2.1(4) we see that

$$
\sum_{i, j<k} \nu_{i j}^{k}\left[\Theta_{i}, \Theta_{j}\right]=\Theta_{k}+G_{k}\left(\Theta_{1}, \ldots, \Theta_{k-1}\right)+H_{k}\left(\Theta_{1}, \ldots, \Theta_{k-1}\right),
$$

where $G_{k}$ and $H_{k}$ are a polynomials over $\mathbb{Q}$ such that $G_{k}\left(\Theta_{1}, \ldots, \Theta_{k-1}\right) \in F_{n_{k}+2} U(\mathfrak{g}, e)$, $H_{k}\left(\Theta_{1}, \ldots, \Theta_{k-1}\right) \in F_{n_{k}} U(\mathfrak{g}, e)$, and $G_{k}$ has zero constant and linear terms. Therefore, $G_{k}\left(\Theta_{1}, \ldots, \Theta_{k-1}\right)$ can be written as a sum of products of elements of $F_{n_{k}+1} U(\mathfrak{g}, e)$ and $H_{k}\left(\Theta_{1}, \ldots, \Theta_{k-1}\right) \in F_{n_{k}+1} U(\mathfrak{g}, e)$. Also, each commutator $\left[\Theta_{i}, \Theta_{j}\right] \in W$, so we have $\Theta_{k} \in W$, as required.

Although Lemma 3.1 shows that we can get by with fewer generators, we in fact use it to show that some relations are not needed for a presentation of $U(\mathfrak{g}, e)$ in Theorem 3.2. The case where $b=r$ in Theorem 3.2 is [18, Lemma 4.1]. Throughout the proof and statement of Theorem 3.2 we use $(\boldsymbol{\Theta})$ as a shorthand for $\left(\Theta_{1}, \ldots, \Theta_{r}\right)$.

Theorem 3.2. Suppose that $\mathfrak{g}^{e}$ is generated by $x_{1}, \ldots, x_{b}$ for $b \leqslant r$. Then $U(\mathfrak{g}, e)$ is generated by $\Theta_{1}, \ldots, \Theta_{r}$ subject only to the relations

$$
\left[\Theta_{i}, \Theta_{j}\right]=F_{i j}\left(\Theta_{1}, \ldots, \Theta_{r}\right)=F_{i j}(\Theta),
$$


for $i=1, \ldots, b$ and $j=1, \ldots, r$, where $F_{i j}$ is a polynomial with coefficients in $\mathbb{Q}$, and $F_{i j}(\Theta) \in F_{n_{i}+n_{j}+2} U(\mathfrak{g}, e)$.

Proof. By Theorem 2.1, the commutator $\left[\Theta_{i}, \Theta_{j}\right]$ is of the form $F_{i j}(\boldsymbol{\Theta})$, where $F_{i j}$ is a polynomial satisfying the stated conditions, for $i, j=1, \ldots, r$. Thanks to [18, Lemma 4.1], $U(\mathfrak{g}, e)$ is generated by the $\Theta_{i}$ subject only to these commutator relations. Therefore, to prove the theorem it suffices to show that the polynomials $F_{k l}(\boldsymbol{\Theta})$ for $k, l=b+1, \ldots, r$ can be determined from the polynomials $F_{i j}(\boldsymbol{\Theta})$ for $i=1, \ldots, b$ and $j=1, \ldots, r$.

As in the proof of Lemma 3.1 , we assume that $x_{b+1}, \ldots, x_{r}$ are chosen so that $n_{b+1} \leqslant \ldots \leqslant n_{r}$ and that

$$
\Theta_{k}=\sum_{i, j<k} \nu_{i j}^{k}\left[\Theta_{i}, \Theta_{j}\right]-G_{k}(\boldsymbol{\Theta})-H_{k}(\boldsymbol{\Theta})
$$

for $k>b$.

Consider $\left[\Theta_{k}, \Theta_{l}\right]$ for some $k, l \in\{b+1, \ldots, r\}$. We assume inductively that we have calculated all $F_{k^{\prime} l^{\prime}}$ for $n_{k^{\prime}}+n_{l^{\prime}}<n_{k}+n_{l}$ in terms of the $F_{i j}$ for $i=1, \ldots, b$ and $j=1, \ldots, r$. We have

$$
\left[\Theta_{k}, \Theta_{l}\right]=\sum_{i, j<k} \nu_{i j}^{k}\left[\left[\Theta_{i}, \Theta_{j}\right], \Theta_{l}\right]-\left[G_{k}(\boldsymbol{\Theta}), \Theta_{l}\right]-\left[H_{k}(\boldsymbol{\Theta}), \Theta_{l}\right]
$$

Consider a term of the form $\left[\left[\Theta_{i}, \Theta_{j}\right], \Theta_{l}\right]$. This commutator has Kazhdan degree $n_{i}+n_{j}+$ $n_{l}+6$ (note that $n_{k}=n_{i}+n_{j}$ ). We can apply the Jacobi identity to obtain

$$
\begin{aligned}
{\left[\left[\Theta_{i}, \Theta_{j}\right], \Theta_{l}\right] } & =\left[\left[\Theta_{i}, \Theta_{l}\right], \Theta_{j}\right]+\left[\Theta_{i},\left[\Theta_{j}, \Theta_{l}\right]\right] \\
& =\left[F_{i l}(\Theta), \Theta_{j}\right]+\left[\Theta_{i}, F_{j l}(\Theta)\right] .
\end{aligned}
$$

By induction, we can determine $F_{i l}(\boldsymbol{\Theta})$ and $F_{j l}(\boldsymbol{\Theta})$ and they both have Kazhdan degree $n_{k}+n_{l}+4$. Now we can apply the Leibniz rule and inductive hypothesis to calculate $\left[F_{i l}(\boldsymbol{\Theta}), \Theta_{j}\right]$ and $\left[\Theta_{i}, F_{j l}(\boldsymbol{\Theta})\right]$ as polynomials in $\boldsymbol{\Theta}$ of Kazhdan degree $n_{k}+n_{l}+2$.

Next consider the term $\left[G_{k}(\boldsymbol{\Theta}), \Theta_{l}\right]$, this has Kazhdan degree $n_{k}+n_{l}+4$, and $G_{k}(\boldsymbol{\Theta})$ has zero linear term. Therefore, we can apply the Leibniz rule and the inductive hypothesis to calculate $\left[G_{k}(\boldsymbol{\Theta}), \Theta_{l}\right]$ as a polynomial in $\boldsymbol{\Theta}$ of Kazhdan degree $n_{k}+n_{l}+2$.

Finally, consider the term $\left[H_{k}(\boldsymbol{\Theta}), \Theta_{l}\right]$. Since $H_{k}(\boldsymbol{\Theta})$ has Kazhdan degree $n_{k}$, this can be calculated as a polynomial in $\boldsymbol{\Theta}$ of Kazhdan degree $n_{k}+n_{l}$ using the Leibniz rule and inductive hypothesis.

Thus we have determined $\left[\Theta_{k}, \Theta_{l}\right]=F_{k l}(\boldsymbol{\Theta})$ as a polynomial of Kazhdan degree $n_{k}+n_{l}+2$, as required.

We next discuss how to find all 1-dimensional representations of $U(\mathfrak{g}, e)$ from a presentation as in Theorem 3.2. From now we fix $b$ such that $x_{1}, \ldots, x_{b}$ generate $\mathfrak{g}^{e}$. Let $\rho: U(\mathfrak{g}, e) \rightarrow \mathbb{C}$ be a 1-dimensional representation of $U(\mathfrak{g}, e)$. Then $\rho$ is determined by the values $\rho\left(\Theta_{i}\right)$ for $i=1, \ldots, r$. These must satisfy the relations $\left[\rho\left(\Theta_{i}\right), \rho\left(\Theta_{j}\right)\right]=F_{i j}\left(\rho\left(\Theta_{1}\right), \ldots, \rho\left(\Theta_{r}\right)\right)$ for $i=1, \ldots, b$ and $j=1, \ldots, r$. We thus see that finding 1-dimensional representations of $U(\mathfrak{g}, e)$ is equivalent to finding solutions to the polynomial equations

$$
F_{i j}\left(t_{1}, \ldots, t_{r}\right)=0 \text { for } i=1, \ldots, b \text { and } j=1, \ldots, r,
$$

with $t_{1}, \ldots, t_{r} \in \mathbb{C}$.

The following lemma and proposition show that the values of certain $t_{i}$ in solutions to (3.3) are forced to be zero, which means that we do not have to consider all the equations in (3.3) to work out all 1-dimensional representations of $U(\mathfrak{g}, e)$. This in turn means that if we only wish to determine the 1-dimensional representations of $U(\mathfrak{g}, e)$, then it is not necessary to calculate all the commutator relations from Theorem 3.2. 
Lemma 3.4. Let $\rho: U(\mathfrak{g}, e) \rightarrow \mathbb{C}$ be a representation of $U(\mathfrak{g}, e)$. Then $\rho\left(\Theta_{i}\right)=0$ for all $i=1, \ldots, r$ such that $\beta_{i} \neq 0$.

Proof. As explained in $\S 2.2$, there is an embedding $\mathfrak{t}^{e} \hookrightarrow U(\mathfrak{g}, e)$ and we identify $\mathfrak{t}^{e}$ with its image in $U(\mathfrak{g}, e)$. Let $i \in\{1, \ldots, r\}$ such that $\beta_{i} \neq 0$ and let $t \in \mathfrak{t}^{e}$ with $\beta_{i}(t) \neq 0$. Then

$$
\beta_{i}(t) \rho\left(\Theta_{i}\right)=\rho\left(\beta_{i}(t) \Theta_{i}\right)=\rho\left(\left[t, \Theta_{i}\right]\right)=\left[\rho(t), \rho\left(\Theta_{i}\right)\right]=0 .
$$

Thus $\rho\left(\Theta_{i}\right)=0$, as required.

The next proposition means that in order to determine the 1-dimensional representations of $U(\mathfrak{g}, e)$, we only need to know the commutators $\left[\Theta_{i}, \Theta_{j}\right]$, when $\beta_{j}=-\beta_{i}$. We need to introduce some notation in order to state the proposition.

Let $I=\left\{i \in\{1, \ldots, r\} \mid \beta_{i}=0\right\}$ and let $J=\left\{(i, j) \in\{1, \ldots, b\} \times\{1, \ldots, r\} \mid \beta_{j}=-\beta_{i}\right\}$. Let $F_{i j}$ be the polynomials from Theorem 3.2 and for $(j, k) \in J$ define $\bar{F}_{j k} \in \mathbb{Q}\left[T_{i} \mid i \in I\right]$ by $\bar{F}_{j k}\left(T_{i} \mid i \in I\right)=F_{j k}\left(\delta_{1} T_{1}, \ldots, \delta_{r} T_{r}\right)$, where $\delta_{i}=1$ if $i \in I$ and $\delta_{i}=0$ if $i \notin I$.

Proposition 3.5. The 1-dimensional representations $\rho: U(\mathfrak{g}, e) \rightarrow \mathbb{C}$ are in bijective correspondence with solutions $\left(t_{i} \mid i \in I\right)$ to the polynomial equations $\bar{F}_{j k}\left(t_{i} \mid i \in I\right)=0$ for $(j, k) \in J$. The solution $\left(t_{i} \mid i \in I\right)$ corresponds to the 1-dimensional representation $\rho$ determined by $\rho\left(\Theta_{i}\right)=t_{i}$ for $i \in I$ and $\rho\left(\Theta_{i}\right)=0$ if $i \notin I$.

Proof. As discussed above, the 1-dimensional representations of $U(\mathfrak{g}, e)$ are given by solutions to (3.3). By Lemma 3.4, we must have $t_{i}=0$ for $i \notin I$ in any solution to these equations. By considering the $\mathfrak{t}^{e}$-weights, we see that each monomial in a polynomial $F_{j k}$ for $(j, k) \notin J$ must contain a $T_{i}$ for some $i \notin I$. The result follows from these two observations.

\section{The algorithm}

In this section we describe our algorithm for calculating a presentation of $U(\mathfrak{g}, e)$ as given in Theorem 3.2. We work with a Chevalley $\mathbb{Z}$-form of $\mathfrak{g}$, and end up with a presentation of $U(\mathfrak{g}, e)$ that is defined over $\mathbb{Z}\left[d^{-1}\right]$, where $d$ is a product of certain primes. We wish to keep $d$ as small as possible, but at some points it is necessary to allow divisions by certain primes, as in the construction in $[\mathbf{1 9}$, Section 2]. Keeping $d$ small allows us to control the bound on $p$ in Theorem 1.2; see Remark 5.2. We keep track of how $d$ 'grows' throughout the algorithm and begin with $d$ being the product of bad primes for $\mathfrak{g}$.

\subsection{Input}

The input to our algorithm is the root system $\Phi$ of a simple Lie algebra $\mathfrak{g}$ over $\mathbb{C}$ along with a set of simple roots $\Pi$ and the labelled Dynkin diagram $D$ of a nilpotent orbit in $\mathfrak{g}$. We write $d_{\alpha}$ for the label of $\alpha \in \Pi$ in $D$.

From this data we can determine the Chevalley $\mathbb{Z}$-form $\mathfrak{g}_{\mathbb{Z}}$ of $\mathfrak{g}$ with Chevalley basis $\left\{h_{\alpha} \mid \alpha \in \Pi\right\} \cup\left\{e_{\alpha} \mid \alpha \in \Phi\right\}$. The Cartan subalgebra $\mathfrak{t}$ has basis $\left\{h_{\alpha} \mid \alpha \in \Pi\right\}$. We define $\mathfrak{g}_{\mathbb{Z}\left[d^{-1}\right]}=\mathfrak{g}_{\mathbb{Z}} \otimes_{\mathbb{Z}} \mathbb{Z}\left[d^{-1}\right]$. The labelled Dynkin diagram $D$ determines a decomposition $\Phi=$ $\bigcup_{j \in \mathbb{Z}} \Phi(j)$, where $\Phi(j)=\left\{\sum_{\alpha \in \Pi} a_{\alpha} \alpha \in \Phi \mid \sum_{\alpha \in \Pi} a_{\alpha} d_{\alpha}=j\right\}$; this in turn gives the grading $\mathfrak{g}=\bigoplus_{j \in \mathbb{Z}} \mathfrak{g}(j)$, where $\mathfrak{g}(j)$ is spanned by the $e_{\alpha}$ with $\alpha \in \Phi(j)$ for $j \neq 0$, and $\mathfrak{g}(0)$ is spanned by $\mathfrak{t}$ and the $e_{\alpha}$ with $\alpha \in \Phi(0)$.

\subsection{Finding the $\mathfrak{s l}_{2}$-triple}

We wish to determine the $\mathfrak{s l}_{2}$-triple $(e, h, f)$. The labelled Dynkin diagram $D$ uniquely determines $h \in \mathfrak{t}$ as follows: we may write $h=\sum_{\alpha \in \Pi} \lambda_{\alpha} h_{\alpha}$, and the $\lambda_{\alpha}$ are uniquely determined 
by the conditions $\sum_{\alpha \in \Pi} \lambda_{\alpha} \beta\left(h_{\alpha}\right)=d_{\beta}$ for all $\beta \in \Pi$. In fact, since the eigenvalues of $h$ on each fundamental irreducible representation of $\mathfrak{g}$ are integers, we have $\lambda_{\alpha} \in \mathbb{Z}_{\geqslant 0}$ for all $\alpha$. We move on to determining $e$. It is well-known that we may choose $e$ to be of the form $e=\sum_{\alpha \in \Gamma} e_{\alpha} \in \mathfrak{g}(2)$, where $\Gamma$ is a certain linearly independent subset of $\Phi(2)$. Now there is a unique $f \in \mathfrak{g}$ such that $(e, h, f)$ is an $\mathfrak{s l}_{2}$-triple. We can find $f$ by writing $f=\sum_{\alpha \in \Phi(2)} \mu_{\alpha} e_{-\alpha}$, then solving for the $\mu_{\alpha}$ in $[e, f]=h$. One can show that it is always possible to choose $e$ so that we get $f \in \mathfrak{g}_{\mathbb{Z}}$ : to do this one reduces inductively to the case where $e$ does not lie in any proper subalgebra of $\mathfrak{g}$ containing $\mathfrak{t}$, and makes an explicit calculation in the remaining cases.

\subsection{Determining the bilinear form}

Now that we have our $\mathfrak{s l}_{2}$-triple we can determine our bilinear form $(\cdot, \cdot)$ on $\mathfrak{g}$. We obtain this by rescaling the Killing form $\kappa$ so that $(e, f)=1$. In general it will not be the case that $(x, y) \in \mathbb{Z}$ for $x, y \in \mathfrak{g}_{\mathbb{Z}} ;$ we require that $(\cdot, \cdot): \mathfrak{g}_{\mathbb{Z}\left[d^{-1}\right]} \times \mathfrak{g}_{\mathbb{Z}\left[d^{-1}\right]} \rightarrow \mathbb{Z}\left[d^{-1}\right]$. Thus we may increase $d$ here to ensure that $\kappa(e, f)$ is invertible in $\mathbb{Z}\left[d^{-1}\right]$. With this choice of bilinear form we have that $\chi \in \mathfrak{g}^{*}$ defined by $\chi(x)=(x, e)$ is such that $\chi: \mathfrak{g}_{\mathbb{Z}\left[d^{-1}\right]} \rightarrow \mathbb{Z}\left[d^{-1}\right]$.

\subsection{Determining the basis}

We next wish to find a basis of $\mathfrak{g}$ as described in $\S 2.3$.

Determining a basis $x_{1}, \ldots, x_{r}$ of $\mathfrak{g}^{e}$ is straightforward, using the Chevalley commutator relations, though we may need to increase $d$ here so that it is a $\mathbb{Z}\left[d^{-1}\right]$-basis of $\mathfrak{g}^{e} \cap \mathfrak{g}_{\mathbb{Z}\left[d^{-1}\right]}$. We order this basis so that $\mathfrak{g}^{e}$ is generated by $x_{1}, \ldots, x_{b}$ and this generating set is chosen so that it is small and convenient to work with. In particular, we want $x_{1}, \ldots, x_{b}$ to generate $\mathfrak{g}^{e} \cap \mathfrak{g}_{\mathbb{Z}\left[d^{-1}\right]}$ over $\mathbb{Z}\left[d^{-1}\right]$, so there is a possibility that we may have to increase $d$ further.

We extend this to get a basis $x_{1}, \ldots, x_{m}$ of $\mathfrak{p}$. We may need to increase $d$ so that $x_{1}, \ldots, x_{m}$ form a $\mathbb{Z}\left[d^{-1}\right]$-basis of $\mathfrak{p}_{\mathbb{Z}\left[d^{-1}\right]}=\mathfrak{g}_{\mathbb{Z}\left[d^{-1}\right]} \cap \mathfrak{p}$.

Next we move on to determine the basis $x_{m+1}, \ldots, x_{m+2 s}$ of $\mathfrak{g}(-1)$. We can choose $x_{m+1}, \ldots, x_{m+s}$ by picking a set of positive roots $\Phi_{+}^{e}$ in $\Phi^{e}$. Then we can readily take $\left\{x_{m+1}, \ldots, x_{m+s}\right\}=\left\{e_{\alpha} \mid \alpha \in \Phi(-1)\right.$ and $\left.\left.\alpha\right|_{\mathfrak{t}^{e}} \in \Phi_{+}^{e}\right\}$. We take each $x_{m+s+i}$ to be a linear combination (over $\mathbb{Q}$ ) of $\left\{e_{\alpha} \mid \alpha \in \Phi(-1)\right.$ and $\left.\left.\alpha\right|_{\mathrm{e}^{e}} \in-\Phi_{+}^{e}\right\}$ so that they are uniquely determined subject to $x_{m+1}, \ldots, x_{m+2 s}$ being a Witt basis of $\mathfrak{g}(-1)$ with respect to $\langle\cdot, \cdot \cdot$. We may have to increase $d$ so that all the coefficients that occur in these linear combinations lie in $\mathbb{Z}\left[d^{-1}\right]$.

To determine our basis $x_{m+2 s+1}, \ldots, x_{m+2 s+s^{\prime}}$ of $\mathfrak{g}(-2)$, we first choose a basis of the kernel of $\chi$ restricted to $\mathfrak{g}(-2)$ to consist of the $e_{-\alpha}$ for $\alpha \in \Phi(2) \backslash \Gamma$, and some elements of the form $e_{-\alpha}+\lambda e_{-\beta}$ for $\alpha, \beta \in \Gamma$ and $\lambda \in \mathbb{Q}$. We extend to a basis of $\mathfrak{g}(-2)$ by adding $f$. We may increase $d$ here to ensure that $\lambda$ lies in $\mathbb{Z}\left[d^{-1}\right]$, thus to ensure that we obtain a $\mathbb{Z}\left[d^{-1}\right]$-basis of

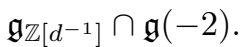

Finally, we extend to a basis $x_{1}, \ldots, x_{n}$ of all of $\mathfrak{g}$ by taking the $x_{i}$ for $i=m+2 s+s^{\prime}+1, \ldots, n$ to be the Chevalley basis elements $e_{\alpha}$ for $\alpha \in \bigcup_{j \leqslant-3} \Phi(j)$.

REMARK 4.1. Although there are many places above where it seems necessary to increase $d$, the cases that we have considered suggest that it suffices to take $d$ to be the product of bad primes for $\mathfrak{g}$ along with those dividing $\kappa(e, f)$. For example, it seems likely that it is always possible to choose $x_{1}, \ldots, x_{m}$ so that $x_{1}, \ldots, x_{r}$ is a $\mathbb{Z}$-basis of $\mathfrak{g}_{\mathbb{Z}} \cap \mathfrak{g}^{e}$, and $x_{1}, \ldots, x_{m}$ is a $\mathbb{Z}$-basis of $\mathfrak{g}_{\mathbb{Z}} \cap \mathfrak{p}$.

\subsection{Finding generators}

We wish to determine expressions for the $\Theta_{i}$ in the form given in Theorem 2.1. First we list the monomials $x^{\mathbf{a}}=x_{1}^{a_{1}} \ldots x_{m+s}^{a_{m+s}}$ for which the coefficient $\lambda_{\mathbf{a}}^{i}$ may be non-zero: according to Theorem 2.1 these are those satisfying $\sum_{j=1}^{m+s} a_{j} \beta_{j}=\beta_{i}$ and $\sum_{j=1}^{m+s} a_{j}\left(n_{j}+2\right) \leqslant n_{i}+2$, 
and excluding those for which either $a_{r+1}=\ldots=a_{m+s}=0$ (that is terms in $U\left(\mathfrak{g}^{e}\right)$ ), or $\sum_{j=1}^{m+s} a_{j}\left(n_{j}+2\right)=n_{i}+2$ and $|\mathbf{a}|=1$ (that is single terms of equal Kazhdan degree to the leading term $\left.x_{i}\right)$.

We choose $K \subseteq\{m+s+1, \ldots, n\}$ so that the nilpotent subalgebra $\mathfrak{m}$ is generated by $\left\{x_{j} \mid j \in K\right\}$; we want to pick $K$ to be small to reduce the amount of computation required. For each $x_{j}$ with $j \in K$, we require $\left[x_{j}, \Theta_{i}\right] \in I_{\chi}$ for $i=1, \ldots, r$. This condition can be calculated using the Chevalley commutator relations and gives rise to a set of linear equations for the coefficients $\lambda_{\mathbf{a}}^{i}$. Solving these equations yields a unique set of coefficients $\lambda_{\mathbf{a}}^{i}$ for $\Theta_{i}$.

In some cases, when $\Theta_{i}$ has large Kazhdan degree, the number of linear equations that we are required to solve is too large for the computation to be feasible. In these cases we try to construct $\Theta_{i}$ in terms of the $\Theta_{j}$ for $j<i$. We chose our basis of $\mathfrak{g}$ so that $\mathfrak{g}^{e}$ is generated by $x_{1}, \ldots, x_{b}$, which allows us to make this construction if $i>b$, where we follow the idea of the proof of Lemma 3.1. In practice, we find an element in $U(\mathfrak{g}, e)$ in terms of the $\Theta_{j}$ with $j<i$ with leading term $x_{i}$ and then subtract monomials in the $\Theta_{j}$ for $j<i$ until the conditions of Theorem 2.1 are met.

The expressions for the generators $\Theta_{i}$ involve coefficients $\lambda_{\mathbf{a}}^{i} \in \mathbb{Q}$. At this point we may need to increase $d$ to ensure that all these coefficients lie in $\mathbb{Z}\left[d^{-1}\right]$.

\subsection{Finding relations}

In order to determine the presentation of $U(\mathfrak{g}, e)$ from Theorem 3.2, we now just have to find the relations. So we have to find polynomials $F_{i j}$ for $i=1, \ldots, b$ and $j=1, \ldots, r$. To find $F_{i j}$ we first evaluate $\left[\Theta_{i}, \Theta_{j}\right]$ as an element of $U(\mathfrak{g})$, that is we take our expressions $\Theta_{i}=u_{i}+I_{\chi}$ and $\Theta_{j}=u_{j}+I_{\chi}$ with $u_{i}, u_{j} \in U(\mathfrak{g})$ and calculate $\left[u_{i}, u_{j}\right]+I_{\chi}$. This determines an expression of the form

$$
\left[\Theta_{i}, \Theta_{j}\right]=\sum_{|\mathbf{a}|_{e} \leqslant n_{i}+n_{j}+2} \mu_{\mathbf{a}}^{i, j} x^{\mathbf{a}}+I_{\chi},
$$

with $\mu_{\mathbf{a}}^{i, j} \in \mathbb{Q}$. Amongst the a with $\mu_{\mathbf{a}}^{i, j} \neq 0$ and $|\mathbf{a}|_{e}$ maximal, there must be one with $a_{k}=0$ for all $k>r$. Let $\mathbf{a}_{1}, \ldots, \mathbf{a}_{c}$ be all such $\mathbf{a}$. Then we consider

$$
\left[\Theta_{i}, \Theta_{j}\right]-\sum_{k=1}^{c} \mu_{\mathbf{a}_{k}}^{i, j} \Theta^{\mathbf{a}_{k}}+I_{\chi} .
$$

This is an element of $U(\mathfrak{g}, e)$ and by construction it must have lower Kazhdan degree than $\left[\Theta_{i}, \Theta_{j}\right]$. We continue by subtracting terms of maximal Kazhdan degree and after a finite number of steps we obtain the required polynomial $F_{i j}$.

In order to have our presentation defined over $\mathbb{Z}\left[d^{-1}\right]$, we may have to increase $d$ so that all coefficients of the polynomials $F_{i j}$ lie in $\mathbb{Z}\left[d^{-1}\right]$.

\subsection{Determining 1-dimensional representations}

Once we have the presentation of $U(\mathfrak{g}, e)$, we can determine all 1-dimensional representations of $U(\mathfrak{g}, e)$ by solving the equations (3.3) or just those from Proposition 3.5. This is achieved using standard techniques for solving polynomial equations.

\subsection{Implementation in GAP}

We have implemented this algorithm in the computer algebra language GAP [8] as explained below.

The Lie algebra $\mathfrak{g}$ is created in GAP by taking the inbuilt Chevalley basis of the simple Lie algebra over $\mathbb{Q}$ and constructing the required basis as explained in $\S$ 4.4. By taking the Lie products in GAP of these elements, we create a table of structure constants from which the 
function LieAlgebraByStructureConstants returns $\mathfrak{g}$ with the required ordered basis, and the universal enveloping algebra $U(\mathfrak{g})$ is created using the function UniversalEnvelopingAlgebra. This allows us to make all of the calculations required in the algorithm.

It should be noted that for calculations and operations in the universal enveloping algebra $U(\mathfrak{g})$ involving elements with many terms GAP functions can be particularly slow and require a lot of memory. Such calculations can be speeded up by storing elements of $U(\mathfrak{g})$ as elements of a particular polynomial ring rather than as elements of the universal enveloping algebra.

\section{Results}

We have used our algorithm to calculate presentations of $U(\mathfrak{g}, e)$ for all cases where $\mathfrak{g}$ is of type $G_{2}, F_{4}$ or $E_{6}$ and $e \in \mathfrak{g}$ is rigid nilpotent. From these presentations we have determined all 1-dimensional representations of $U(\mathfrak{g}, e)$. For $\mathfrak{g}$ of type $E_{7}$ and $e$ rigid nilpotent, we have calculated all generators of $U(\mathfrak{g}, e)$ and enough relations to determine all 1-dimensional representations of $U(\mathfrak{g}, e)$ using Proposition 3.5.

In these cases we found that there are one or two 1-dimensional representations of $U(\mathfrak{g}, e)$, as shown in Table 1 . Here we give the Bala-Carter label of the rigid nilpotent orbits in $\mathfrak{g}$; the column indicates whether the number of 1-dimensional representations of $U(\mathfrak{g}, e)$ is one or two. A list of rigid orbits can be found in [21, p. 173].

REMARK 5.1. For some cases where $\mathfrak{g}$ is of type $E_{8}$ and $e \in \mathfrak{g}$ is rigid nilpotent we are able to show that there exist 1-dimensional representations of $U(\mathfrak{g}, e)$. We have checked this when $e$ has Bala-Carter label $A_{1}, 2 A_{1}, 3 A_{1}, 4 A_{1}, A_{2}+A_{1}, A_{2}+2 A_{1}, A_{2}+3 A_{1}, 2 A_{2}+A_{1}$ and $A_{3}+A_{1}$, where in each but the last case there is just one 1-dimensional representation while in the last instance there are two such representations. At present it is computationally unfeasible to deal with the remaining eight rigid nilpotent $e \in \mathfrak{g}$.

Recall that the height of $e$ is defined to be the maximal $j$ for which $\mathfrak{g}(j) \neq 0$. When the height of $e$ is large then there are generators $\Theta_{i}$ of $U(\mathfrak{g}, e)$ with large Kazhdan degree. Consequently, the expression for $\Theta_{i}$ given in Theorem 2.1 can be very complicated. This means that it is at present unfeasible to determine all generators, and to calculate the required commutators.

REMARK 5.2. We discuss some rationality issues related to Theorem 1.2. In our algorithm we keep track of a positive integer $d$ such that $U(\mathfrak{g}, e)$ is defined over $\mathbb{Z}\left[d^{-1}\right]$. For $\mathfrak{g}$ of type $G_{2}$, $F_{4}$ or $E_{6}$ and $e$ rigid, where we have calculated a full presentation of $U(\mathfrak{g}, e)$, we see that we can take $d$ to be the product of the bad primes for $\mathfrak{g}$ along with those dividing $\kappa(e, f)$. This means that the reduction modulo $p$ argument from [19, Section 2] goes through for all good $p$ not dividing $\kappa(e, f)$. This allows one to obtain an explicit lower bound $M$ on $p$ for Theorem 1.2 for $\mathfrak{g}$ of type $G_{2}, F_{4}$ or $E_{6}$, that is so that the conclusions of the theorem hold for all $p>M$. This bound $M$ is determined by considering all pairs $\left(\mathfrak{l}, e_{0}\right)$, where $\mathfrak{l}$ is a Levi subalgebra of $\mathfrak{g}$ and $e_{0} \in \mathfrak{l}$ is rigid nilpotent, then taking $M$ to be the maximum prime dividing $\kappa_{\mathfrak{l}}\left(e_{0}, f_{0}\right)$, where $\left(e_{0}, h_{0}, f_{0}\right)$ is an $\mathfrak{s l}_{2}$-triple in $\mathfrak{l}$ and $\kappa_{\mathfrak{l}}$ is the Killing form on $\mathfrak{l}$. To ensure that this is the correct bound it was necessary to calculate presentations of the finite $W$-algebras $U\left(\mathfrak{l}, e_{0}\right)$ associated to all such pairs $\left(\mathfrak{l}, e_{0}\right)$. Explicitly, for $G_{2}$ we obtain $M=3$, and for $F_{4}$ and $E_{6}$ we

TABLE 1. The number of 1-dimensional representations of $U(\mathfrak{g}, e)$ for e rigid.

\begin{tabular}{ccc}
\hline $\mathfrak{g}$ & 1 & 2 \\
\hline$G_{2}$ & $A_{1}$ & $\widetilde{A}_{1}$ \\
$F_{4}$ & $A_{1}, \widetilde{A}_{1}, A_{1}+\widetilde{A}_{1}, A_{2}+\widetilde{A}_{1}$ & $\widetilde{A}_{2}+A_{1}$ \\
$E_{6}$ & $A_{1}, 3 A_{1}, 2 A_{2}+A_{1}$ & \\
$E_{7}$ & $A_{1}, 2 A_{1},\left(3 A_{1}\right)^{\prime}, 4 A_{1}, A_{2}+2 A_{1}, 2 A_{2}+A_{1}$ & $\left(A_{3}+A_{1}\right)^{\prime}$ \\
\hline
\end{tabular}


obtain $M=5$. We have to omit the prime 5 for $\mathfrak{g}$ of type $F_{4}$ or $E_{6}$ as it divides $\kappa\left(e_{0}, f_{0}\right)$, where $e_{0}$ is a certain rigid nilpotent element for $\mathfrak{l}$ of type $B_{3}$ or $D_{5}$ respectively.

For the cases where $\mathfrak{g}$ is of type $E_{7}$ it is not possible to be so explicit about a bound. We have not calculated all the relations, and we cannot rule out the possibility that the other relations will lead to an increase in $d$. In all the relations that we have calculated the only primes occurring in denominators of coefficients are bad primes for $\mathfrak{g}$, so it seems likely that this is the case for all relations. If this were true, then we would get an analogous bound as for $\mathfrak{g}$ in the previous paragraph.

\section{An example}

We illustrate our algorithm with an explicit example where $\mathfrak{g}$ is of type $G_{2}$ and $e$ is a short root vector.

Let $\mathfrak{g}$ be the simple Lie algebra of type $G_{2}$. Then GAP gives the Chevalley basis $b_{1}, \ldots, b_{14}$, where $b_{1}, \ldots, b_{6}$ denote the positive root vectors, $b_{7}, \ldots, b_{12}$ denote the negative root vectors, and the 2-dimensional Cartan subalgebra is generated by $b_{13}=\left[b_{1}, b_{7}\right]$ and $b_{14}=\left[b_{2}, b_{8}\right]$. We divide the Killing form by 24 to obtain our bilinear form $(\cdot, \cdot)$.

An $\mathfrak{s l}_{2}$-triple for the orbit is determined to be $e=b_{4}, h=2 b_{13}+3 b_{14}$ and $f=b_{10}$. We construct our basis $x_{1}, \ldots, x_{14}$ in terms of the Chevalley basis $b_{1}, \ldots, b_{14}$ as described in $\S$ 4.4. In Table 2 we give this basis and show which parts of the basis form bases of $\mathfrak{g}^{e}, \mathfrak{p}$ and $\mathfrak{m}$, respectively. We also give the values of $n_{i}$ and $\beta_{i}$; as $\mathfrak{t}^{e}=\mathbb{C} x_{6}$ is 1 -dimensional, the weight $\beta_{i}$ can be identified with the integer such that $\left[x_{6}, x_{i}\right]=\beta_{i} x_{i}$. We note that we can pick $x_{1}, \ldots, x_{m}$ to be multiples of elements of the Chevalley basis; this is not the case in general. We observe that our basis is a $\mathbb{Z}\left[\frac{1}{2}\right]$-basis of $\mathfrak{g}_{\mathbb{Z}\left[\frac{1}{2}\right]}$; however, we only view it as a $\mathbb{Z}\left[\frac{1}{6}\right]$-basis of $\mathfrak{g}_{\mathbb{Z}\left[\frac{1}{6}\right]}$ as we have already divided by 3 to make $(e, f)=1$ (also 3 is a bad prime for $G_{2}$ ). We note that there is no small generating set of $\mathfrak{g}^{e}$, so we take $b=r=6$; also there is no small generating set of $\mathfrak{m}$ so we take $K$ from $\S 4.5$ to be $\{11,12,13,14\}$.

We calculate $\Theta_{i}$ as explained in $\S$ 4.5. We illustrate the procedure with the calculation of $\Theta_{1}$. We have that $\Theta_{1}$ is of the form $\Theta_{1}=\left(x_{1}+\sum \lambda_{\mathbf{a}}^{1} x^{\mathbf{a}}\right)+I_{\chi}$ and satisfies the conditions of Theorem 2.1. We first determine for which a the coefficient $\lambda_{\mathbf{a}}^{1}$ can be non-zero and denote this set by $A_{1}$; the elements of $A_{1}$ correspond to the monomials $x_{4} x_{6} x_{10}, x_{4} x_{7}, x_{4} x_{9} x_{10}, x_{4} x_{10}$, $x_{6} x_{8}, x_{8}$ and $x_{8} x_{9}$. We next calculate $\left[x_{i}, x_{1}+\sum_{\mathbf{a} \in A_{1}} \lambda_{\mathbf{a}}^{1} x^{\mathbf{a}}\right]$ in $U(\mathfrak{g})$ for $i=11, \ldots, 14$, viewing the $\lambda_{\mathbf{a}}^{1}$ as indeterminates. Then we project into $Q_{\chi}=U(\mathfrak{g}) / I_{\chi}$ to obtain a set of linear equations to solve for the coefficients $\lambda_{\mathrm{a}}^{1}$. These equations have a unique solution which determines the value of $\Theta_{1}$. We determine $\Theta_{i}$ for $i=2, \ldots, 6$ in the same way and obtain the complete list of generators of $U(\mathfrak{g}, e)$ :

$$
\begin{aligned}
\Theta_{1}= & \left(x_{1}+3 x_{4} x_{6} x_{10}+x_{4} x_{7}+2 x_{4} x_{9} x_{10}-4 x_{4} x_{10}\right. \\
& \left.+2 x_{6} x_{8}-4 x_{8}+x_{8} x_{9}\right)+I_{\chi} \\
\Theta_{2}= & \left(x_{2}+\frac{1}{2} x_{3} x_{10}-\frac{3}{2} x_{4} x_{5} x_{10}+\frac{1}{2} x_{4} x_{10}^{3}-x_{5} x_{8}-x_{6} x_{7}\right. \\
& -\frac{1}{2} x_{6} x_{9} x_{10}+x_{6} x_{10}+2 x_{7}-x_{7} x_{9}+\frac{1}{2} x_{8} x_{10}^{2} \\
& \left.+\frac{5}{2} x_{9} x_{10}-\frac{1}{2} x_{9}^{2} x_{10}-3 x_{10}\right)+I_{\chi}
\end{aligned}
$$

\begin{tabular}{|c|c|c|c|c|c|c|c|c|c|c|c|c|c|c|}
\hline & \multicolumn{9}{|c|}{$\mathfrak{p}$} & \multirow[b]{3}{*}{$x_{10}$} & \multicolumn{4}{|c|}{$\mathfrak{m}$} \\
\hline & \multicolumn{6}{|c|}{$\mathfrak{g}^{e}$} & \multirow[b]{2}{*}{$x_{7}$} & \multirow[b]{2}{*}{$x_{8}$} & \multirow[b]{2}{*}{$x_{9}$} & & \multirow{2}{*}{$x_{11}$} & \multirow[b]{2}{*}{$x_{12}$} & \multirow[b]{2}{*}{$x_{13}$} & \multirow[b]{2}{*}{$x_{14}$} \\
\hline & $\overline{x_{1}}$ & $x_{2}$ & $x_{3}$ & $x_{4}$ & $x_{5}$ & $x_{6}$ & & & & & & & & \\
\hline & $b_{6}$ & $b_{5}$ & $b_{4}$ & $b_{2}$ & $b_{8}$ & $b_{14}$ & $b_{1}$ & $b_{3}$ & $b_{13}$ & $b_{9}$ & $\frac{1}{2} b_{7}$ & $b_{10}$ & $b_{11}$ & $b_{12}$ \\
\hline$n_{i}$ & 3 & 3 & 2 & 0 & 0 & 0 & 1 & 1 & 0 & -1 & -1 & -2 & -3 & -3 \\
\hline$\beta_{i}$ & 1 & -1 & 0 & 2 & -2 & 0 & -1 & 1 & 0 & -1 & 1 & 0 & 1 & -1 \\
\hline
\end{tabular}

TABLE 2. Basis of $\mathfrak{g}$ of type $G_{2}$. 


$$
\begin{aligned}
& \Theta_{3}=\left(x_{3}+\frac{3}{4} x_{4} x_{10}^{2}+3 x_{6} x_{9}+x_{8} x_{10}-5 x_{9}+x_{9}^{2}\right)+I_{\chi} \\
& \Theta_{4}=x_{4}+I_{\chi} \\
& \Theta_{5}=\left(x_{5}-\frac{1}{4} x_{10}^{2}\right)+I_{\chi} \\
& \Theta_{6}=x_{6}+I_{\chi} .
\end{aligned}
$$

Next we calculate the relations. We illustrate this by calculating the polynomial $F_{3,5}$ such that $\left[\Theta_{3}, \Theta_{5}\right]=F_{3,5}\left(\Theta_{1}, \ldots, \Theta_{6}\right)$. We begin by taking the expressions for $\Theta_{3}$ and $\Theta_{5}$ above and calculating

$$
\left[\Theta_{3}, \Theta_{5}\right]=\left(9 x_{5} x_{6}-\frac{9}{4} x_{6} x_{10}^{2}-\frac{51}{2} x_{5}+\frac{15}{8} x_{10}^{2}\right)+I_{\chi} .
$$

The monomial $x_{5} x_{6}$ is the only monomial of Kazhdan degree $n_{3}+n_{5}+2$ and consists of basis elements of $\mathfrak{g}^{e}$ that occur in the above expression. Therefore, according to the method described in $\S 4.6$, we consider

$$
\left[\Theta_{3}, \Theta_{5}\right]-9 \Theta_{5} \Theta_{6}=\left(-\frac{51}{2} x_{5}-\frac{51}{8} x_{10}^{2}\right)+I_{\chi} .
$$

Following the algorithm leads us to calculate

$$
\left[\Theta_{3}, \Theta_{5}\right]-9 \Theta_{5} \Theta_{6}+\frac{51}{2} \Theta_{5}=0+I_{\chi}
$$

Therefore, we have calculated the relation and we have that $F_{3,5}\left(T_{1}, \ldots, T_{6}\right)=9 T_{5} T_{6}-\frac{51}{2} T_{5}$.

The other commutator relations are found in the same way and all are given below; we omit all commutators that are equal to 0 :

$$
\begin{aligned}
{\left[\Theta_{1}, \Theta_{2}\right]=} & 5 \Theta_{3} \Theta_{4} \Theta_{5}-\frac{1}{2} \Theta_{3}^{2}-\Theta_{3} \Theta_{6}^{2}+9 \Theta_{4} \Theta_{5} \Theta_{6}^{2} \\
& -\frac{9}{2} \Theta_{4}^{2} \Theta_{5}^{2}+7 \Theta_{3} \Theta_{6}-\frac{69}{2} \Theta_{4} \Theta_{5} \Theta_{6}+6 \Theta_{6}^{3} \\
& -6 \Theta_{3}+\frac{93}{4} \Theta_{4} \Theta_{5}-30 \Theta_{6}^{2}+42 \Theta_{6}-18 \\
{\left[\Theta_{1}, \Theta_{3}\right]=} & 6 \Theta_{1} \Theta_{6}-3 \Theta_{2} \Theta_{4}-3 \Theta_{1} \\
{\left[\Theta_{1}, \Theta_{5}\right]=} & \Theta_{2} \\
{\left[\Theta_{1}, \Theta_{6}\right]=} & -\Theta_{1} \\
{\left[\Theta_{2}, \Theta_{3}\right]=} & -3 \Theta_{1} \Theta_{5}-6 \Theta_{2} \Theta_{6}+12 \Theta_{2} \\
{\left[\Theta_{2}, \Theta_{4}\right]=} & \Theta_{1} \\
{\left[\Theta_{2}, \Theta_{6}\right]=} & \Theta_{2} \\
{\left[\Theta_{3}, \Theta_{4}\right]=} & -9 \Theta_{4} \Theta_{6}+\frac{15}{2} \Theta_{4} \\
{\left[\Theta_{3}, \Theta_{5}\right]=} & 9 \Theta_{5} \Theta_{6}-\frac{51}{2} \Theta_{5} \\
{\left[\Theta_{4}, \Theta_{5}\right]=} & \frac{1}{2}+\Theta_{6} \\
{\left[\Theta_{4}, \Theta_{6}\right]=} & -2 \Theta_{4} \\
{\left[\Theta_{5}, \Theta_{6}\right]=} & 2 \Theta_{5} .
\end{aligned}
$$

We can observe that 2 is the only prime occurring in the denominators in the formulas for the generators and relations. Therefore, the finite $W$-algebra $U(\mathfrak{g}, e)$ is defined over $\mathbb{Z}\left[\frac{1}{6}\right]$.

We move on to determining the 1-dimensional representations of $U(\mathfrak{g}, e)$. That is, we have to find the solutions to the equations (3.3) where the polynomials $F_{i j}$ are given in the commutator relations above. We immediately see that we have

$$
t_{1}=t_{2}=t_{4}=t_{5}=0 \quad \text { and } \quad t_{6}=-\frac{1}{2} .
$$

We substitute these values in $F_{1,2}$ and are left to solve

$$
-\frac{1}{2} t_{3}^{2}-\frac{39}{4} t_{3}-\frac{189}{4}=0
$$

for $t_{3}$. This gives the solutions $t_{3}=-9,-\frac{21}{2}$, so there are two 1 -dimensional representations of $U(\mathfrak{g}, e)$. 
Acknowledgements. We are grateful to A. Premet for suggesting the problem considered in this paper and for various helpful conversations. The last author acknowledges the financial support of the EPSRC. Part of this paper was written during a stay of the authors at the Isaac Newton Institute for Mathematical Sciences, Cambridge, during the 'Algebraic Lie Theory' Programme in 2009. We thank the referee for some useful comments.

\section{References}

1. J. Brundan and S. M. Goodwin, 'Good grading polytopes', Proc. London Math. Soc. (3) 94 (2007) $155-180$.

2. J. Brundan, S. M. Goodwin and A. Kleshchev, 'Highest weight theory for finite $W$-algebras', Int. Math. Res. Not. 15 (2008) Art. ID rnn051.

3. J. Brundan and A. Kleshchev, 'Representations of shifted Yangians and finite $W$-algebras', Mem. Amer. Math. Soc. 196 (2008).

4. A. D'Andrea, C. De Concini, A. De Sole, R. Heluani and V. Kac, 'Three equivalent definitions of finite $W$-algebras', appendix to [5].

5. A. De Sole and V. Kac, 'Finite vs affine $W$-algebras', Jpn. J. Math. 1 (2006) 137-261.

6. E. M. Friedlander and B. J. Parshall, 'Modular representation theory of Lie algebras', Amer. J. Math. 110 (1988) no. 6, 1055-1093.

7. W. L. Gan and V. Ginzburg, 'Quantization of Slodowy slices', Int. Math. Res. Not. 5 (2002) 243-255.

8. The GAP GrouP, 'GAP-groups, algorithms, and programming, version 4.3', 2002, http://www.gap-system.org.

9. V. Ginzburg, 'Harish-Chandra bimodules for quantized Slodowy slices', Represent. Theory 13 (2009) $236-271$.

10. I. Losev, 'Quantized symplectic actions and W-algebras', J. Amer. Math. Soc. 23 (2010) no. 1, 35-59.

11. I. Losev, 'Finite dimensional representations of W-algebras', Preprint, 2008, arXiv:0807.1023.

12. I. Losev, 'On the structure of the category $\mathcal{O}$ for $W$-algebras', Preprint, 2008, arXiv:0812.1584.

13. I. Losev, ' 1-dimensional representations and parabolic induction for $W$-algebras', Preprint, 2009, arXiv:0906.0157.

14. G. Lusztig and N. Spaltenstein, 'Induced unipotent classes', J. London Math. Soc. (2) 19 (1979) 41-52.

15. A. Premet, 'Irreducible representations of Lie algebras of reductive groups and the Kac-Weisfeiler conjecture', Invent. Math. 121 (1995) 79-117.

16. A. Premet, 'Special transverse slices and their enveloping algebras', Adv. Math. 170 (2002) 1-55.

17. A. Premet, 'Enveloping algebras of Slodowy slices and the Joseph ideal', J. Eur. Math. Soc. 9 (2007) $487-543$.

18. A. Premet, 'Primitive ideals, non-restricted representations and finite $W$-algebras', Mosc. Math. J. 7 (2007) 743-762.

19. A. Premet, 'Commutative quotients of finite W-algebras', Preprint, 2008, arXiv:0809.0663.

20. S. Skryabin, 'A category equivalence', appendix to [16].

21. N. Spaltenstein, Classes unipotentes et sous-groupes de Borel, Lecture Notes in Mathematics 946 (Springer, New York, 1982).

\author{
Simon M. Goodwin \\ School of Mathematics \\ University of Birmingham \\ Birmingham, B15 2TT \\ United Kingdom \\ goodwin@maths.bham.ac.uk \\ Glenn Ubly \\ School of Mathematics \\ University of Southampton \\ Southampton, SO17 1BJ \\ United Kingdom \\ gu@soton.ac.uk
}

\author{
Gerhard Röhrle \\ Fakultät für Mathematik \\ Ruhr-Universität Bochum \\ D-44780 Bochum \\ Germany \\ gerhard.roehrle@rub.de
}

